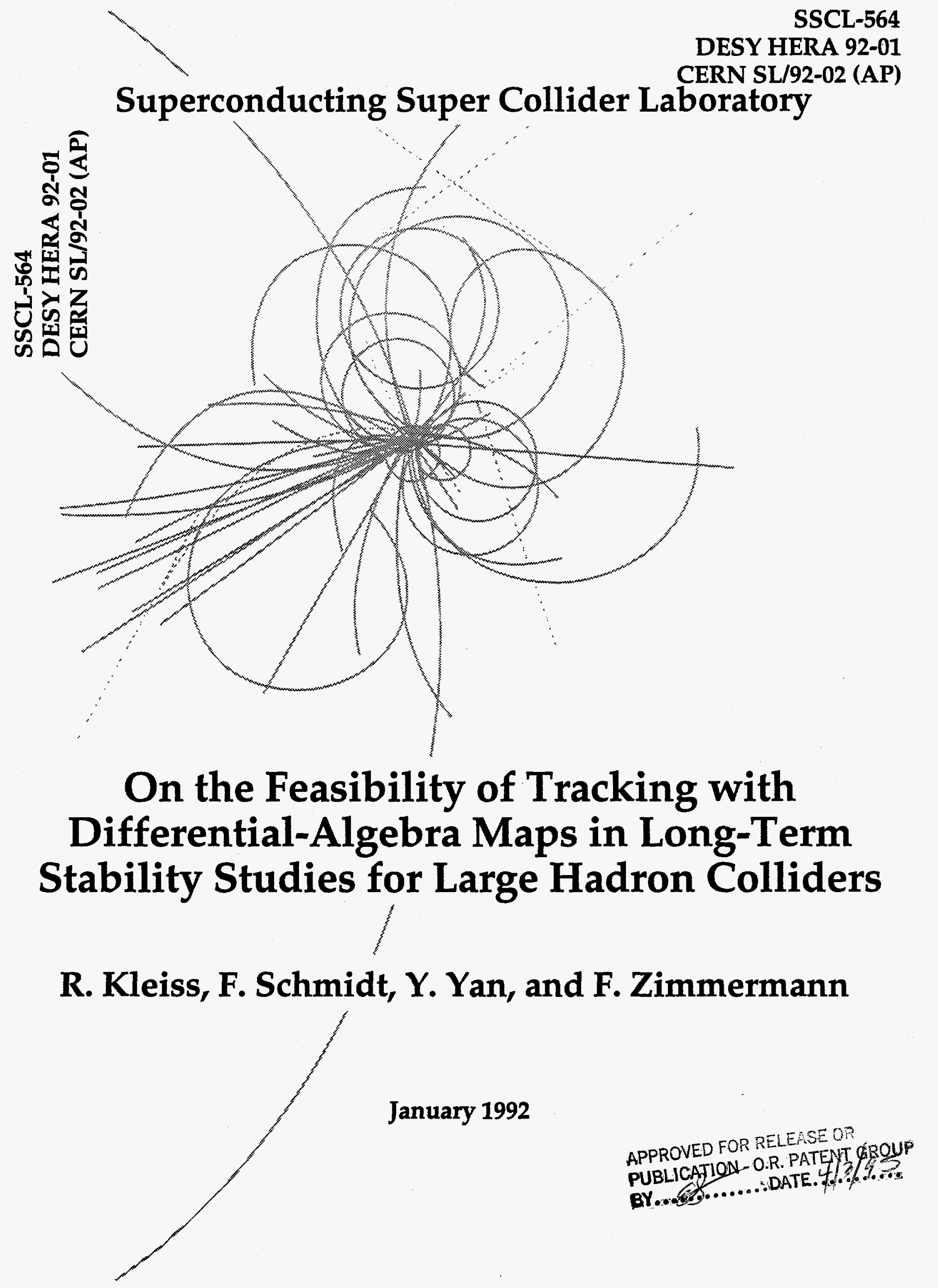


SSCL-564

DESY HERA 92-01

CERN SL/92-02 (AP)

On the Feasibility of Tracking with Differential-Algebra Maps in Long-Term Stability Studies for Large Hadron Colliders

\author{
R. Kleiss \\ TH Division \\ CERN \\ Geneva, Switzerland \\ F. Schmidt \\ SL Division \\ CERN \\ Geneva, Switzerland \\ Y. Yan
}

Accelerator Design and Operations Division

Superconducting Super Collider Laboratory*

2550 Beckleymeade Avenue

Dallas, Texas 75237

F. Zimmermann

DESY

Hamburg, Germany

January 1992

"Operated by the Universities Research Association, Inc., for the U.S. Department of Energy under Contract No. DE-AC35-89ER40486. 



\section{DISCLAIMER}

Portions of this document may be illegible in electronic image products. Images are produced from the best available original document. 
SSCL-564

DESY HERA 92-01

CERN SL/92-02 (AP)

\title{
On the Feasibility of Tracking with Differential-Algebra Maps in Long-Term Stability Studies for Large Hadron Colliders
}

\author{
R. Kleiss, F. Schmidt, Y. Yan, and F. Zimmermann
}

\begin{abstract}
A time-saving alternative to conventional element-by-element tracking in long-term stability studies is the use of truncated Taylor maps. This report discusses how the nonsymplecticity of a moderately high-order truncated Taylor map affects its reliability when the map is used for tracking over several thousand turns. Various machines and two different map-constructing programs are compared. It is found that the discrepancies between the Taylor map results and those obtained by direct tracking grow with amplitude. Thus, such maps are not guaranteed to be sufficient for long-term tracking over millions of turns without suitable symplectification.
\end{abstract}

\section{DISCLAIMER}

This report was prepared as an account of work sponsored by an agency of the United States Government. Neither the United States Government nor any agency thereof, nor any of their employees, makes any warranty, express or implied, or assumes any legal liability or responsibility for the accuracy, completeness, or usefulness of any information, apparatus, product, or process disclosed, or represents that its use would not infringe privately owned rights. Reference herein to any specific commercial product, process, or service by trade name, trademark, manufacturer, or otherwise does not necessarily constitute or imply its endorsement, recommendation, or favoring by the United States Government or any agency thereof. The views and opinions of authors expressed herein do not necessarily state or reflect those of the United States Government or any agency thereof. 



\section{CONTENTS}

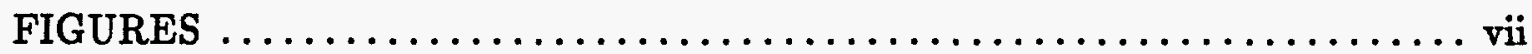

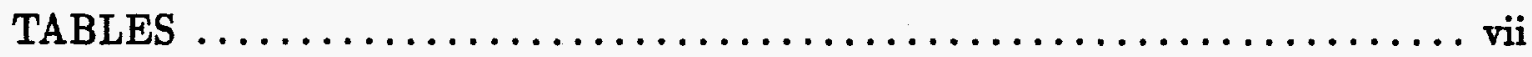

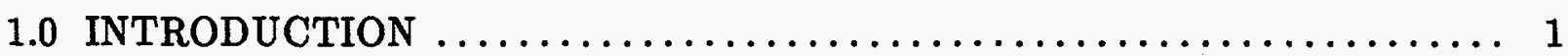

2.0 THE MODELS UNDER CONSIDERATION $\ldots \ldots \ldots \ldots \ldots \ldots \ldots \ldots \ldots$

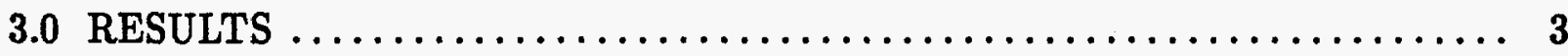

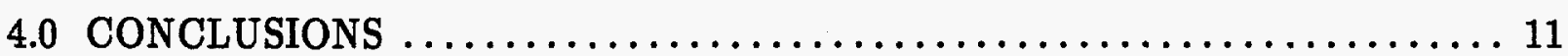

ACKNOWLEDGEMENTS $\ldots \ldots \ldots \ldots \ldots \ldots \ldots \ldots \ldots \ldots \ldots \ldots \ldots \ldots$

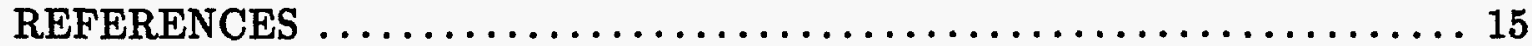


, 


\section{FIGURES}

1. One-turn Accuracies for Various Initial Amplitudes and Orders of the

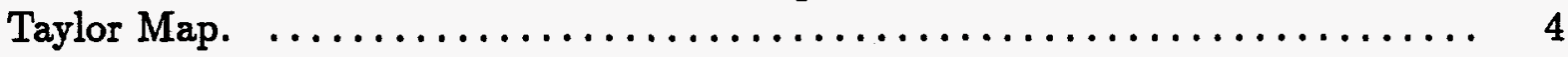

2. One-turn Error as a Function of Turn Number. $\ldots \ldots \ldots \ldots \ldots \ldots \ldots \ldots \ldots .5$

3. Accumulated Amplitude Error as a Function of Turn Number. ........... 6

4. Absolute Value of the Relative Amplitude Error Per Turn as a Function of Amplitude. $\ldots \ldots \ldots \ldots \ldots \ldots \ldots \ldots \ldots \ldots \ldots \ldots \ldots \ldots \ldots \ldots, 8$

5. Amplitude Evolution as a Function of Turn Number for the LHC. ........ 9

6. Relative Amplitude Error as a Function of Turn Number for the SSC. . . . . . 10

7. Amplitude Evolution as a Function of Turn Number for the LHC. . . . . . . 11

8. Amplitude Evolution as a Function of Turn Number for the SSC. $\ldots \ldots \ldots \ldots .12$

\section{TABLES}

1. Amplitudes and Average Deviation Increase Per Turn for the Total Transverse Amplitude $A_{x y}$ for the $\mathrm{LHC}(\mathrm{N}), \mathrm{LHC}(\mathrm{R}), \mathrm{LHC}(\mathrm{T})$, and SSC, for Various Initial Amplitudes.

2. Stability Behavior for Direct and DA Tracking, for the LHC With and Without Random Errors. 


\subsection{INTRODUCTION}

Large proton storage rings such as HERA, the Large Hadron Collider (LHC), or the Superconducting Super Collider (SSC) suffer from significant and unavoidable magnet nonlinearities. Extensive computer simulations are necessary to investigate the problem of long-term stability in these colliders. In the conventional approach trajectories of many particles are followed over millions of turns, element-by-element through the accelerator structure, using tracking codes that take into account only the effect of external forces. In view of restricted computer resources, a time-saving method has become highly desirable. This report deals with an apparently promising alternative. It tries to answer the question of whether a complete storage ring consisting of thousands of magnets can be replaced by a moderately high-order polynomial map in a simulation, without losing information about the dynamic aperture.

In recent years it has repeatedly been argued that the Taylor maps obtained by analyzing standard tracking codes like SIXTRACK ${ }^{1}$ or TEAPOT ${ }^{2}$ with differential-algebra (DA) techniques ${ }^{3}$ might be used to perform actual tracking studies. A $7^{\text {th }}$-order map of the SSC from after the interaction region to before the interaction region has been suggested ${ }^{4}$ as being sufficient for the SSC beam-beam studies. A study of beam survival as a function of initial amplitude and turn number $N$ was performed ${ }^{5}$ for the SSC using an $11^{\text {th }}$-order map; good agreement was reported between DA- and direct-tracking (DI) results for $N \leq 10^{6}$.

However, such survival results give only a rough global indication of the beam behavior. In some cases, one would also like to be able to make more detailed, long-term studies, such as the divergence of nearby trajectories (and the onset of chaotic motion).

In particular, due to the truncation at finite order, DA (Taylor) maps are inherently not symplectic, and it would be extremely surprising if there were no trace of this nonsymplecticity in evidence. Of course, by increasing the order of the map, the nonsymplecticity may be made arbitrarily small. But as the map size grows exponentially, DA-tracking decelerates quickly with increasing order, and a $14^{\text {th }}$-order map is (for the LHC) expected to be no faster than direct tracking using SIXTRACK. Alternatively, one could imagine restoring symplecticity by modifying the Taylor map. However, this requires further investigation.

For phase-space trajectories inside the dynamic aperture, the amplitudes should be constant when averaged over a suitably large number of turns (1000, for instance). This is borne out by direct-tracking simulations using SIXTRACK, which treats the dynamics in a symplectic* manner. For all cases that we examined, the DI results show perfectly constant

\footnotetext{
"Apart from the effects of rounding errors.
} 
average amplitudes. Deviations from symplecticity in the DA approach may therefore be expected to show up as a time (turn number) dependence of the average amplitude.

It should be stressed that we have examined several different maps: one produced for the SSC using TEAPOT, ${ }^{2}$ one produced for the LHC also using TEAPOT, and one set of maps produced for the LHC with SIXTRACK. ${ }^{1}$ We have studied the amplitude evolution using both DI and DA, for four different cases, denoted as LHC(N), LHC(R), LHC(T), and SSC (see below).

\subsection{THE MODELS UNDER CONSIDERATION}

We have constructed $11^{\text {th }}$-order Taylor maps for an LHC with the following characteristics: the nominal LHC lattice with interaction zones and nonlinear chromaticity and amplitude-dependent tune shift corrections with sextupoles, octupoles, and decapoles à la Neuffer. In one version of the map, $(N)$, there were systematic dipole errors but no random dipole errors; in another version, $(\mathrm{R})$, random dipole errors were also included. In no case have we considered quadrupole and skew errors or closed-orbit corrections.

The production of the $11^{\text {th }}$-order map using SIXTRACK ${ }^{1}$ and the available DA packages ${ }^{3,6}$ takes about 3 CPU hours on the IBM (about 1 CPU hour on the CRAY); the map-tracking procedure is about 10 times faster than the analogous direct-tracking program. We also used a map produced from the equivalent program TEAPOT, ${ }^{2}$ which, due to its splitting each element into several thin elements and due to the complex frame transfer between thin elements for better dispersion calculation, takes about 20 CRAY hours for the same $\mathrm{LHC}$ lattice. This version is denoted by $\mathrm{LHC}(\mathrm{T})$; it contains no random errors. (Since random errors are implemented in a different way in SIXTRACK and TEAPOT, this would make comparison difficult.) In addition we have investigated an SSC map that was obtained by analyzing the program TEAPOT and that took about 10 CPU hours on the CRAY. We regret that we did not consider an SSC map from SSCTRK, which would take about 0.3 CPU hours on CRAY for an $11^{\text {th }}$-order SSC map.

We have also constructed a hybrid map in which the cavity is treated exactly, as in DI, and the rest of the machine with DA. Since the DA map has to deal with only five variables, the resulting code is about twice as fast as full DA-tracking; no appreciable differences are evident in the results.

The phase-space coordinates of the particles are denoted in the transverse part by $x, x^{\prime}, y$, and $y^{\prime}$, and in the longitudinal part by $z, z^{\prime}$, where the primes denote the momenta. These are understood to be Courant-Snyder transformed, which makes them independent of the $\beta$ functions. In addition to the Euclidean phase-space distance between two points, we have also studied various amplitudes: $A_{x}=\sqrt{x^{2}+x^{\prime 2}}, A_{y}=\sqrt{y^{2}+y^{\prime 2}}, A_{z}=\sqrt{z^{2}+z^{12}}$, 
$A_{x y}=\sqrt{A_{x}^{2}+A_{y}^{2}}$, and $A_{x y z}=\sqrt{A_{x y}^{2}+A_{z}^{2}}$, which we then average over a number of turns in order to separate the global, long-term evolution from short-term fluctuations.

Our reason for disregarding the phase information is that although the correlation in phase between DI- and DA-tracking results is expected to deteriorate rapidly for even small $N$, this is not a worry in practice as long as the absolute amplitudes remain well-behaved. The quantity we are concerned with in this paper is the time evolution of the average amplitudes $A$, as a function of the initial amplitude.

\subsection{RESULTS}

A first point of interest is how well DA reproduces DI on the local level of one turn. In Figure 1 we present the "one-turn accuracy" as defined in Reference 5-that is, the phasespace distance between a point obtained from DA and one from DI after one turn, starting at the same initial point. For the $\mathrm{LHC}(\mathrm{N})$ case, we compare different orders of the map and various initial amplitudes; we have also added analogous results for the $L H C(T)$ and the SSC. For both map-producing programs, the one-turn accuracy is seen to be essentially the same. As expected, the agreement increases with the order of the map and decreases with increasing initial amplitude. Note, however, that the saturation of accuracy for the SSC, at about $10^{-8}$, requires further investigation. The very good agreement between the two different maps-LHC(N) and LHC(T)-for the LHC, and the essential agreement between the two TEAPOT-produced maps for LHC and SSC, give us confidence in our ability to construct maps correctly and as accurately as DA will allow.

The differences between DI and DA seen in Figure 1 are of limited practical relevance because a part of the phase-space distance is a difference in phase, which we are quite willing to accept as long as the absolute amplitudes are well-behaved. Therefore we have performed the following exercise: we follow the DI evolution, predicting at each turn the result of the next turn using DA, and then comparing that result with the DI result. In this case we have disregarded the phase information and we compare only the values of $A_{x y}$ (which is 0.5835 for DI in this case). The differences are plotted in Figure 2, for LHC(N). The main feature of this plot is an oscillation over 5 orders of magnitude in the predictive power of DA at twice the synchrotron frequency. We ascribe this to the fact that the dependence of the transverse degrees of freedom on the relative momentum deviation is more important than that on the path-length difference. If the momentum deviation happens to be small (twice per synchrotron oscillation), the error is also reduced.

The real object of interest is, of course, not how well DA predicts the evolution on the time scale of one turn but, rather, over many turns. In Figure 3, therefore, we have plotted, for the same machine and initial amplitudes as before, the accumulated difference in $A_{x y}$ 
between the DI- and DA-tracking results. In analogy with Figure 2, a modulating effect of the synchrotron oscillations can be seen: whenever the momentum deviation becomes large, the difference between DI and DA increases, resulting in a stepwise increase in the accumulated error. For this initial amplitude, the overall picture is that of a linear increase in amplitude.

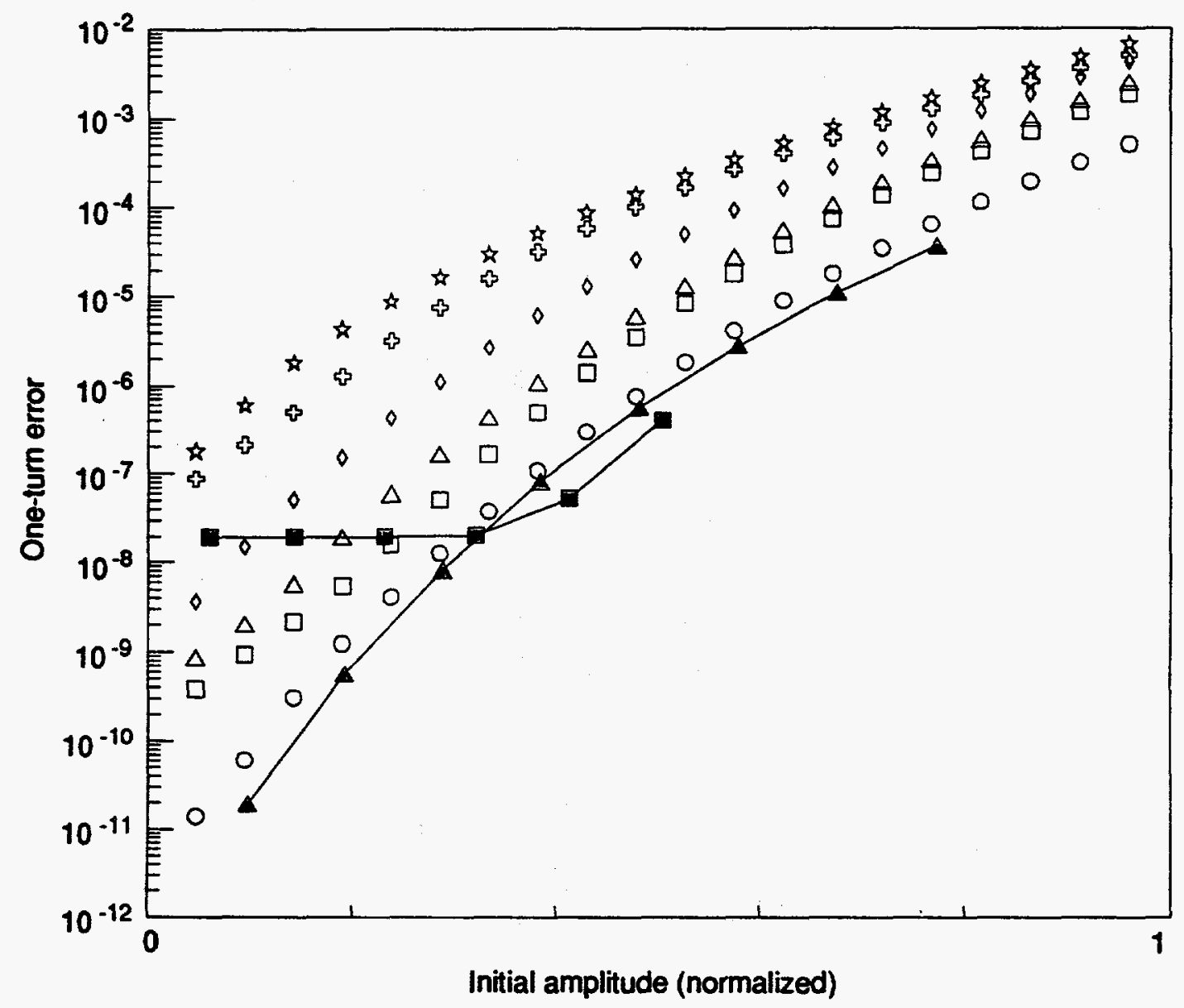

Figure 1. One-turn Accuracies for Various Initial Amplitudes and Orders of the Taylor Map. Open symbols refer to LHC(N), with map order from 11 (circles) down to 6 (stars). Closed symbols refer to $\mathrm{LHC}(\mathrm{T})$ (triangles) and SSC (squares), both for $11^{\text {th }}$-order maps. At the dynamic aperture, the $11^{\text {th }}$-order one-turn errors are $2 \times 10^{-4}$ for the $L H C(N)$ (initial amplitude $=0.88$ ), $3 \times 10^{-5}$ for the $L H C(T)$ (initial amplitude $=0.77$ ), and $3 \times 10^{-8}$ for the SSC (initial amplitude $=0.42$ ).

It is also useful to gain an impression of the overall behavior of the deviations between DI and DA. To this end, we computed for $N$ turns the average values $A_{i}^{D I}$ and $A_{i}^{D A}$ for $\mathrm{DI}$ and DA, respectively, with $i=x, y, z, x y, x y z$. The average amplitude deviation per turn, $\delta A_{i}$, is then defined as $\delta A_{i}=\left(A_{i}^{D A}-A_{i}^{D I}\right) /\left(N A_{i}^{D I}\right)$. In Table 1 , we give the results for our four cases: $\mathrm{LHC}(\mathrm{N}), \mathrm{LHC}(\mathrm{R}), \mathrm{LHC}(\mathrm{T})$, and SSC(R). We took $N=20,000$ for the LHC versions and $N=10,000$ for the SSC. The different models $L H C(N), L H C(R)$, and $\mathrm{LHC}(\mathrm{T})$ are seen to be in general agreement with one another, while the results for the 
SSC are in agreement with those for the LHC where the initial amplitudes overlap. As expected, $\delta A_{x y}$ increases with increasing initial amplitude. In particular, for the $L H C(N)$ and $L H C(R)$, some particles with large initial amplitudes (indicated in the table) were lost after a smaller number of turns. These losses are purely artifacts of the DA approach, since for DI tracking all these amplitudes are stable. On the basis of these results, one might have already concluded that an $11^{\text {th }}$-order DA tracking is not reliable for the LHC since some particles near (but still inside) the dynamic aperture have already gotten lost within 12,000 turns. As for the SSC, the smaller amplitude deviation $\delta_{x y}=5.3 \times 10^{-9}$ at the dynamic aperture $A_{x y}=0.42$ (3 to 4 orders of magnitudes smaller compared with those of the $\mathrm{LHC}$ at the dynamic aperture) is probably the reason that the one-million-turn survival plots from the $11^{\text {th }}$-order map tracking and from the element-by-element tracking agree globally. ${ }^{5}$ However, it should be noted that there is no a priori way of judging whether such an $11^{\text {th }}$-order SSC map can be used for even longer $\left(>10^{6}\right)$ turn tracking without checking the results afterwards.

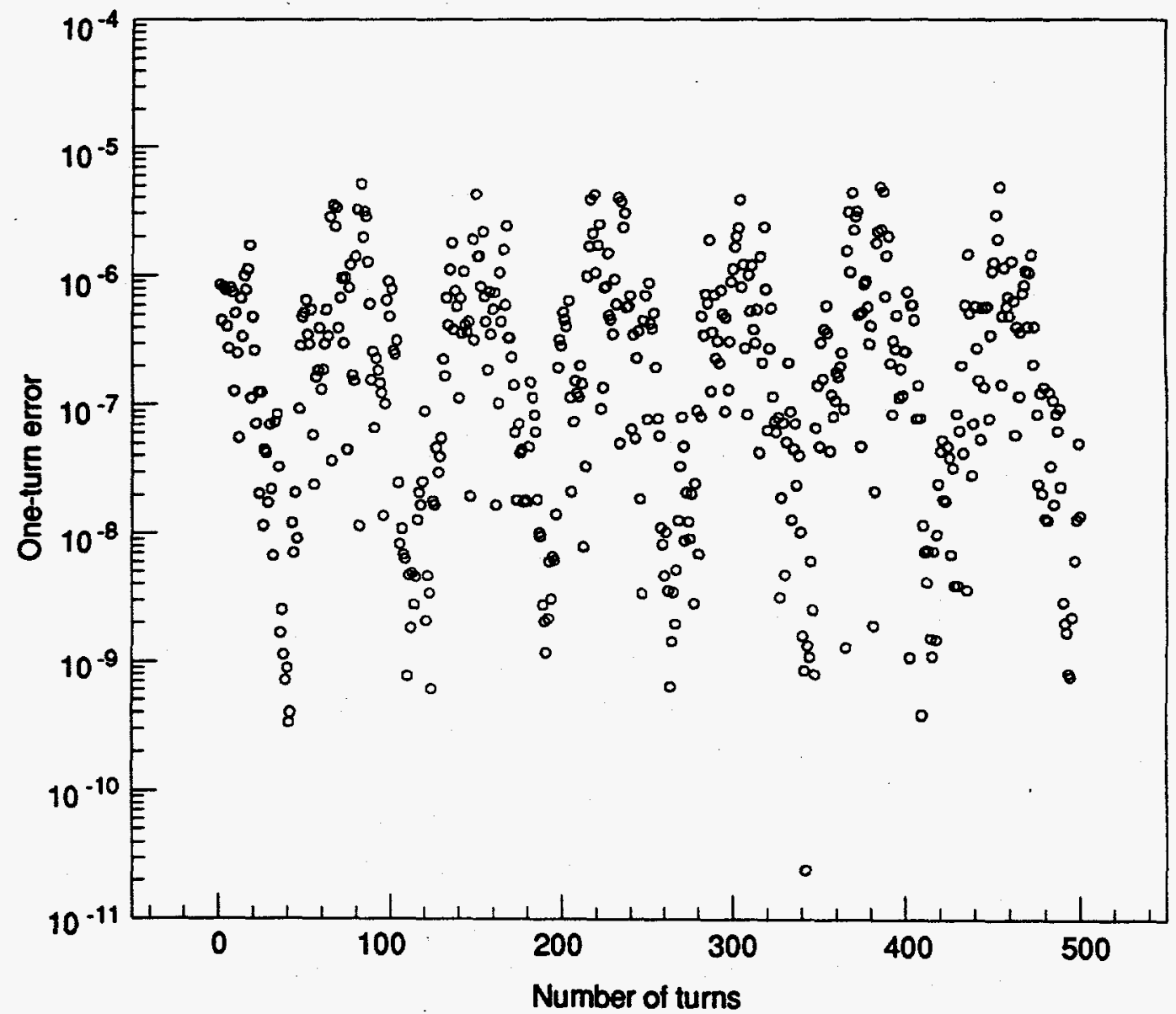

Figure 2. One-turn Error as a Function of Turn Number. The variations in the error over 5 orders of magnitude have twice the frequency of the synchrotron oscillation. 


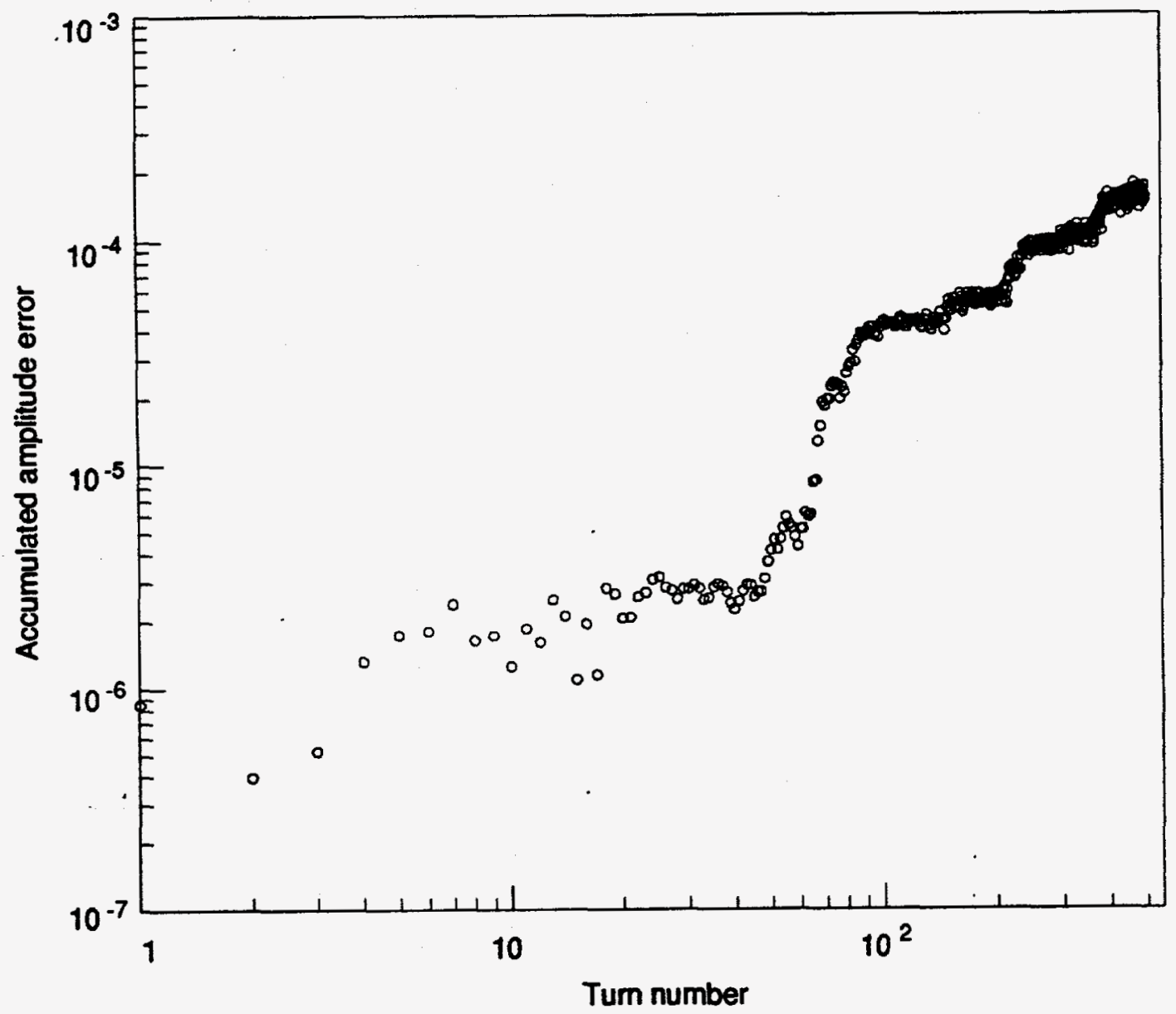

Figure 3. Accumulated Amplitude Error as a Function of Turn Number. The amplitude error grows approximately linearly with time, and the step-like increases are a reflection of the oscillations in the one-turn error evident in Figure 2.

Figure 4 is a graphical representation of Table 1 . It shows the absolute value of the relative amplitude error per turn as a function of amplitude. For all amplitudes up to the dynamic aperture, the error is approximately the same in all four cases. We conclude that the causes for these errors are solely related to the order of the map and not to the specific model under investigation. Figure 4 can therefore be used, with some care, to evaluate the error that is to be expected for other models and to estimate a maximum number of turns for which DA can be usefully applied, depending on one's opinion of what is an acceptable accumulated error. For comparison, the corresponding relative averaged amplitude decrease per turn for SIXTRACK due to computer roundoff errors is estimated ${ }^{7}$ to be about $2 \times 10^{-13}$ at an amplitude of $A_{x}=3.0$, which is three times the maximum amplitude depicted in Figure 4! It should be noted that even in the case of LHC with no random errors, the particles with initial amplitude of 0.779 are already lost after 12,000 turns, while DI tracking shows that the actual dynamic aperture in that case is a good deal larger (see Table 2). 
TABLE 1. AMPLITUDES AND AVERAGE DEVIATION INCREASE PER TURN FOR THE TOTAL TRANSVERSE AMPLITUDE $A_{x y}$ FOR THE LHC(N), LHC(R), LHC(T), AND SSC, FOR VARIOUS INITIAL AMPLITUDES.

\begin{tabular}{|c|c|c|c|c|c|c|c|}
\hline \multicolumn{2}{|c|}{ LHC(N) } & \multicolumn{2}{c|}{ LHC(R) } & \multicolumn{2}{c|}{ LHC(T) } & \multicolumn{2}{c|}{ SSC } \\
\hline$A_{x y}$ & $\delta A_{x y}$ & $A_{x y}$ & $\delta A_{x y}$ & $A_{x y}$ & $\delta A_{x y}$ & $A_{x y}$ & $\delta A_{x y}$ \\
\hline & & & & & & 0.0595 & $5.76 \mathrm{E}-11$ \\
0.0971 & $1.36 \mathrm{E}-11$ & 0.0972 & $1.80 \mathrm{E}-11$ & 0.0982 & $1.83 \mathrm{E}-11$ & & \\
0.1942 & $6.85 \mathrm{E}-11$ & 0.1941 & $9.91 \mathrm{E}-11$ & 0.1950 & $7.62 \mathrm{E}-11$ & & \\
0.2914 & $8.70 \mathrm{E}-10$ & 0.2907 & $1.04 \mathrm{E}-9$ & 0.2918 & $8.90 \mathrm{E}-10$ & & \\
& & & & & & $0.3262^{*}$ & $-1.29 \mathrm{E}-9$ \\
0.3887 & $8.32 \mathrm{E}-9$ & 0.3870 & $9.09 \mathrm{E}-9$ & 0.3887 & $8.32 \mathrm{E}-9$ & & \\
& & & & & & 0.4178 & $5.31 \mathrm{E}-9$ \\
0.4861 & $5.58 \mathrm{E}-8$ & $0.4830^{*}$ & $5.69 \mathrm{E}-8$ & 0.4861 & $5.51 \mathrm{E}-8$ & & \\
& & & & & & $0.5056^{*}$ & $9.26 \mathrm{E}-6$ \\
0.5835 & $2.92 \mathrm{E}-7$ & 0.5781 & $2.74 \mathrm{E}-7$ & 0.5830 & $2.79 \mathrm{E}-7$ & & \\
0.6813 & $1.38 \mathrm{E}-6$ & 0.6745 & $1.21 \mathrm{E}-6$ & 0.6803 & $-6.05 \mathrm{E}-5$ & & \\
$0.7791^{(1)}$ & $8.27 \mathrm{E}-6$ & $0.7716^{(2)}$ & $9.27 \mathrm{E}-6$ & 0.7779 & $-1.00 \mathrm{E}-5$ & & \\
$0.8283^{(3)}$ & $1.55 \mathrm{E}-5$ & $0.8119^{(4)}$ & $1.05 \mathrm{E}-5$ & & & & \\
$0.8779^{*}$ & $-9.70 \mathrm{E}-6$ & $0.8628^{(3)}$ & $1.95 \mathrm{E}-5$ & & & & \\
$0.9242^{(6)}$ & $5.26 \mathrm{E}-5$ & $0.9104^{(7)}$ & $5.71 \mathrm{E}-5$ & & & & \\
\hline
\end{tabular}

Note: Where indicated by superscripts the particle was lost in DA after (1):12,000, (2):10,000, (3):6000, (4):8000, (5):3000, (6):1400, and (7):1800 turns. Entries with an asterisk are discussed in greater detail in the text. The dynamic aperture for the SSC is $A_{2 y}=0.42$; the dynamic aperture for the LHC with random errors is $A_{x y}=0.77$, and without random errors is $A_{x y}=0.88$.

A more important observation to be made is that the differences between DI and DA tracking do not appear to lend themselves to easy analysis. To illustrate this point we present in Figures 5 to 8 different examples of amplitude evolution.

The first example, Figure 5, is $A_{x y}$ for the LHC, with random errors and an initial amplitude of 0.483 . An increase is evident; on our time scale of 20,000 turns it appears linear. Other, quite similar, initial amplitudes for the SSC give a linear decrease. This is 


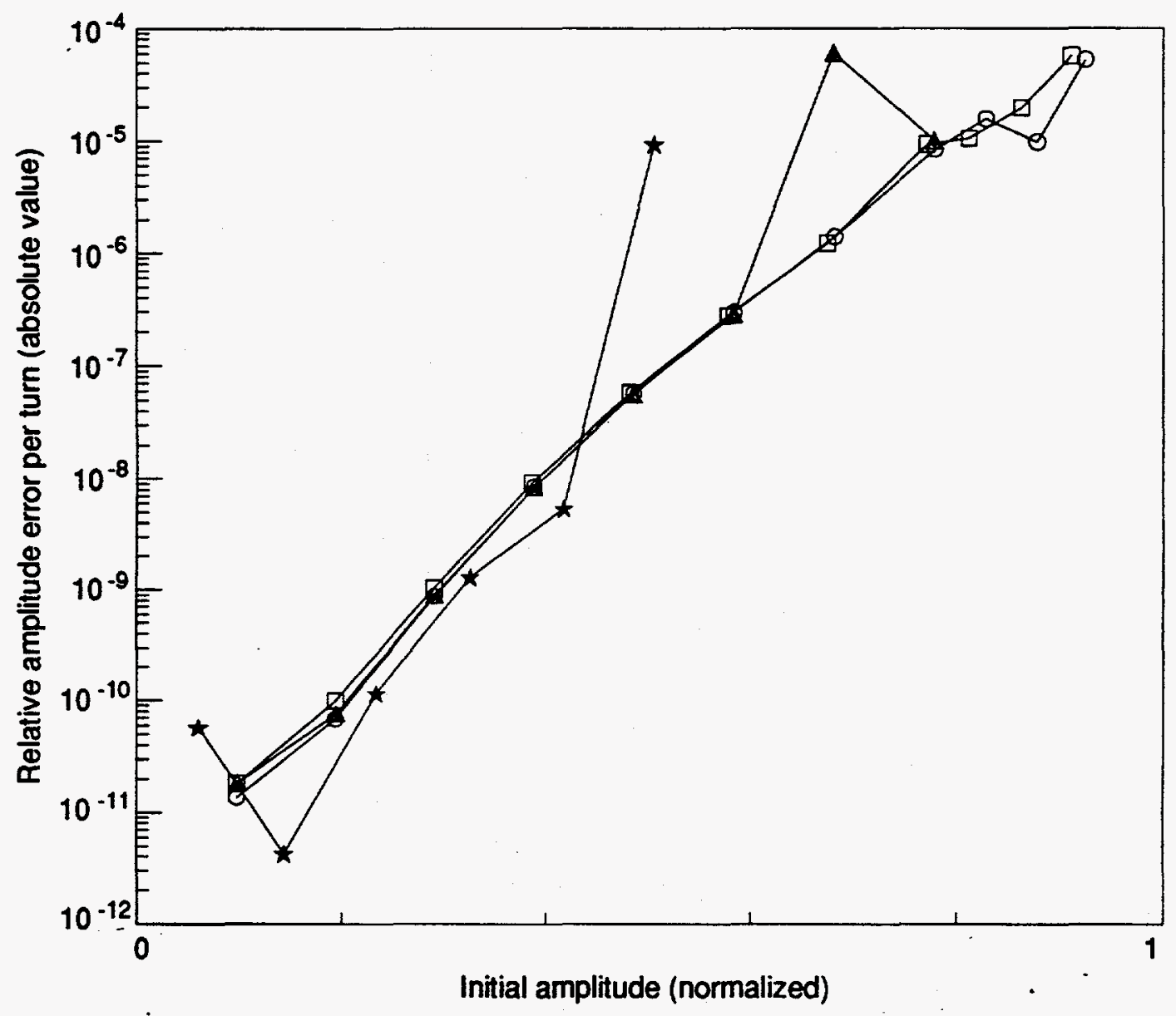

Figure 4. Absolute Value of the Relative Amplitude Error Per Turn as a Function of Amplitude. The circles, squares, triangles, and stars represent the results for LHC(N), LHC(R), LHC(T) and SSC, respectively. The fact that the amplitude errors in all four cases are approximately the same leads us to conclude that the errors are due to the order of the map only. The graph can therefore be used to estimate the errors for other tracking models.

TABLE 2. STABILITY BEHAVIOR FOR DIRECT AND DA TRACKING, FOR THE LHC WITH AND WITHOUT RANDOM ERRORS.

\begin{tabular}{|c|c|c|c|l|}
\hline \multicolumn{2}{|c|}{ LHC(N) } & \multicolumn{2}{c|}{ LHC(R) } & \multicolumn{2}{c|}{. Description } \\
\hline DI & DA & DI & DA & \\
\hline 0.878 & 0.681 & & 0.675 & $\begin{array}{l}\text { false onset of chaos in DA } \\
\text { onset of chros } \\
\text { false particle loss in DA } \\
\text { particle loss before 20,000 turns }\end{array}$ \\
\hline
\end{tabular}

Note: For each case the initial amplitude at which qualitatively new behavior occurs is entered. 
the (undesirable but regular) behavior one expects from a map with a small symplecticity violation.

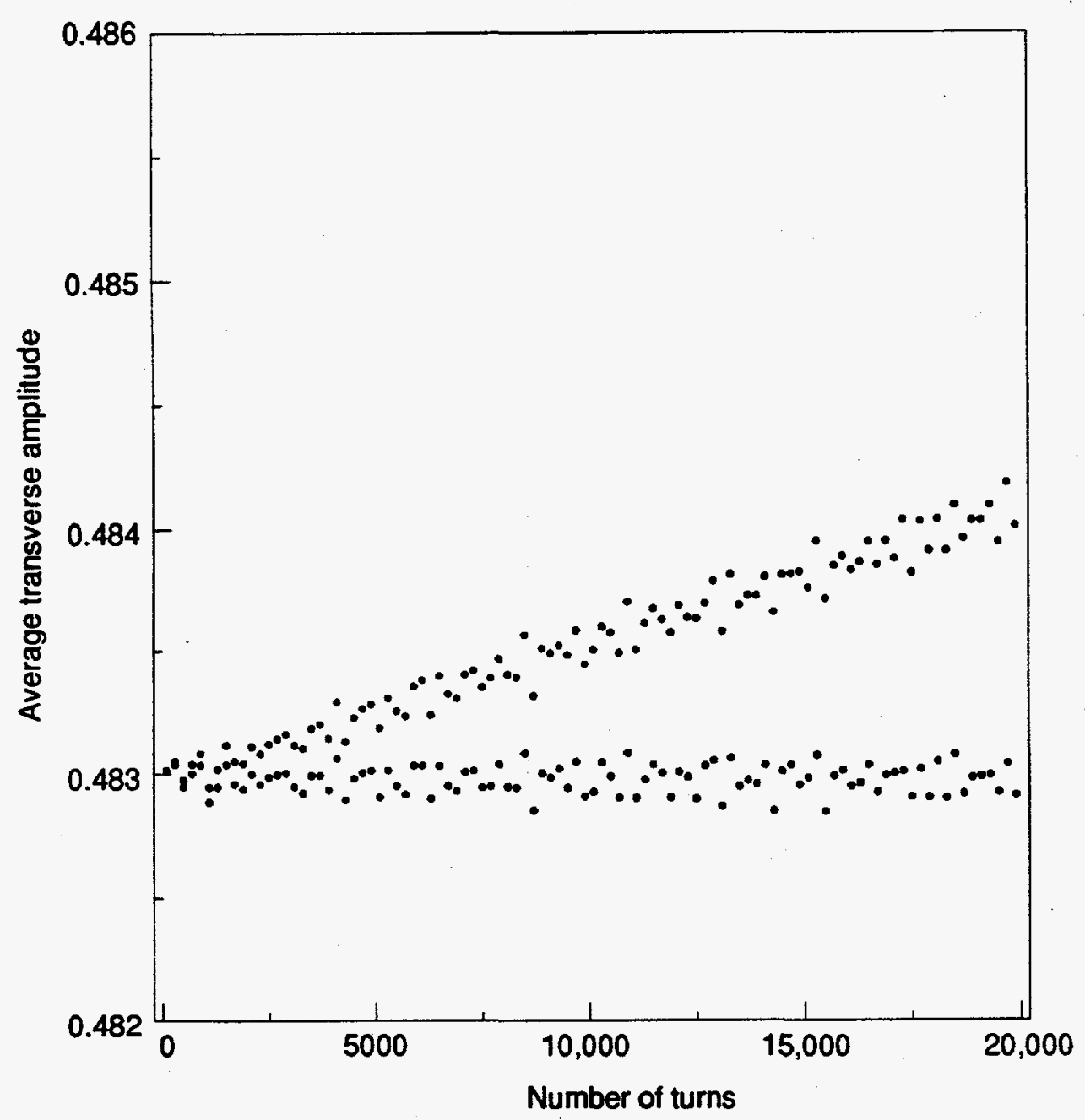

Figure 5. Amplitude Evolution as a Function of Turn Number for the LBC. With direct tracking, the amplitude is perfectly constant; with map tracking, the amplitude grows approximately linearly with time. This behavior is typical for not-too-large initial amplitudes and for our $11^{\text {th }}$-order map.

In Figure 6 we give an example for the SSC with an initial amplitude of 0.326 , where on the average the amplitude is decreasing. On top of that, however, we find that the amplitude oscillates more and more rapidly.

Figure 7 shows a most bizarre case for the LHC, with no random errors at an initial amplitude of 0.878 . At first the amplitude increases drastically, and the particle appears to be lost after some 3500 turns. However, the trajectory miraculously recovers, and the transverse amplitude $A_{x y}$ starts decreasing steadily. It should be noted that this behavior falls in the middle of the region, which under DA is unstable; for amplitudes 0.779 and 0.924 , the particle is simply lost after 6000 and 1400 turns, respectively. 


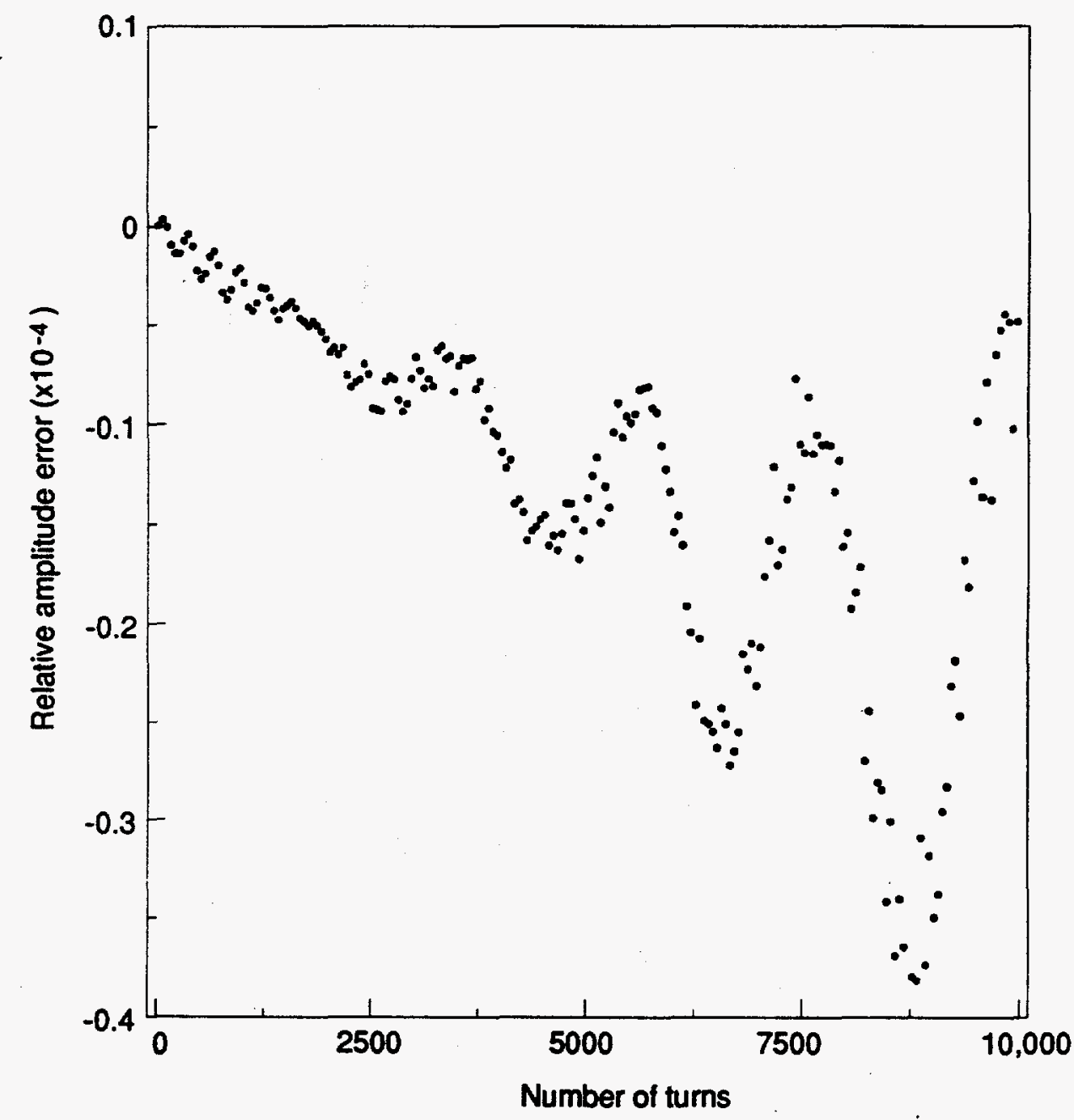

Figure 6. Relative Amplitude Error as a Function of Turn Number for the SSC. In the case of the SSC, we typically find a decrease of the DA amplitude compared to direct tracking. Due to large oscillations in the direct-tracking amplitude, we present here the relative difference of the amplitudes $A_{x y}^{D A}$ and $A_{x y}^{D I}$ each averaged over 50 turns.

Finally, in Figure 8 we present the results for the SSC with an initial amplitude of 0.506 . Note that this amplitude is outside of the dynamic aperture (0.42). Here we find that the DA prediction, after faithfully following the DI results for some 2000 turns, suddenly exhibits a rapid growth of amplitude.

One of the most interesting parameters in the long-term stability studies is the Lyapunov exponent, which describes the transition from the regular to the chaotic regime. ${ }^{8}$ This can be studied by tracking two particles with a small difference in initial coordinates. We calculated this phase-space distance for DI and DA tracking, and determined the regularity or chaoticity of the time evolution.

In Table 2 we present the different predictions of stability in DA and DI, for both $\mathrm{LHC}(\mathrm{N})$ and LHC(R). In each case, the amplitude at which chaotic motion appears is 
largely underestimated by some 15-20\% in DA tracking. The same holds for estimates of amplitudes where particle loss starts to occur before the $20,000^{\text {th }}$ turn.

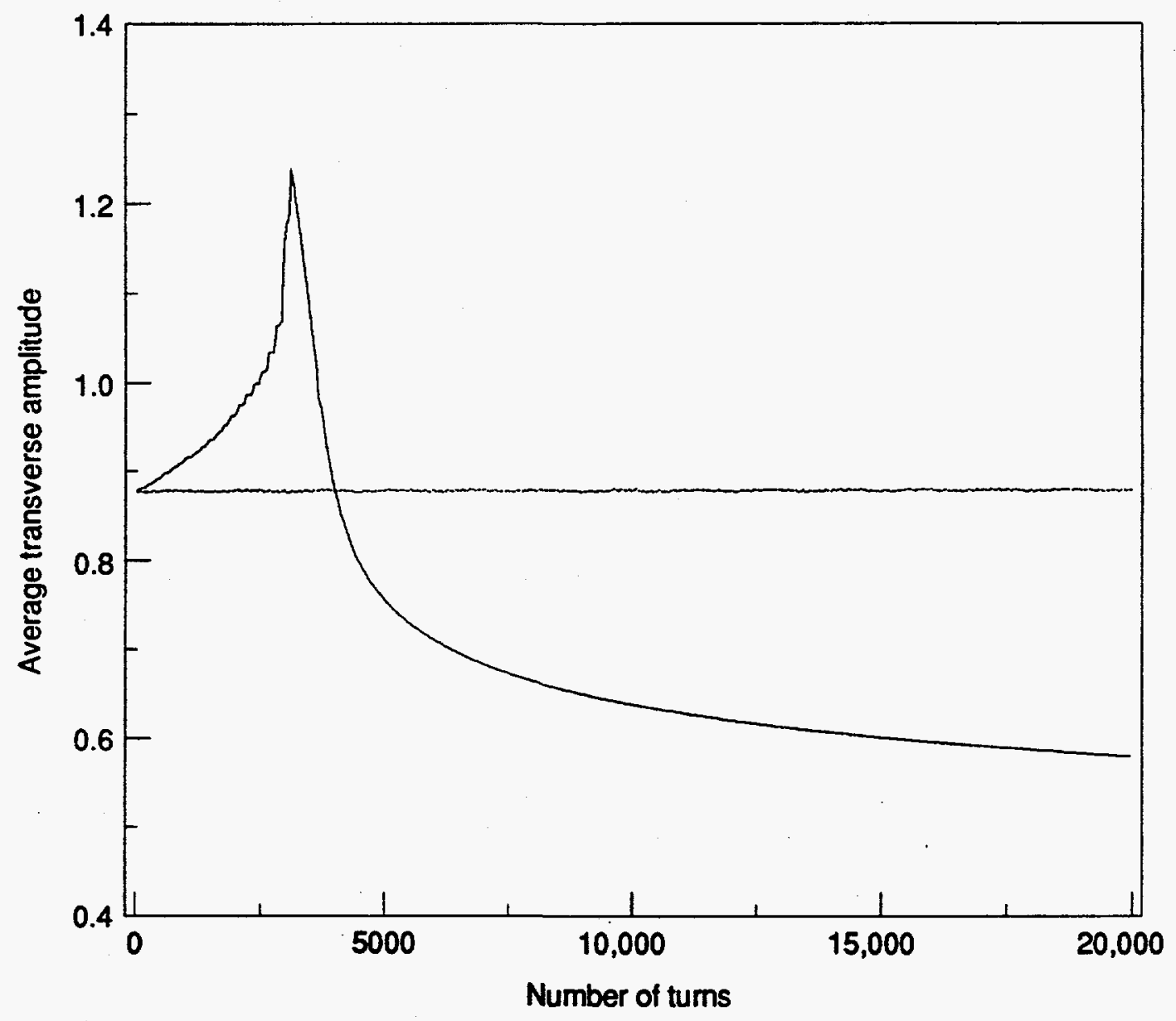

Figure 7. Amplitude Evolution as a Function of Turn Number for the LHC. For large amplitudes (in the chaotic regime) the direct-tracking amplitude is still a constant over this time range. The DA amplitude, after an initial rapid growth, recovers miraculously and starts to settle down to zero. Note that this amplitude falls in the middle of a range where in DA tracking the particle is artificially lost after a small number of turns.

\subsection{CONCLUSIONS}

We have compared direct and $11^{\text {th }}$-order DA tracking for typical LHC and SSC layouts. We observed that because of insufficient symplecticity, DA tracking leads to unpredictable deviations from the expected behavior of the trajectories. We have observed both increases and decreases in amplitude, increasingly rapid oscillations, and cusp-like behavior. Moreover, the same map may display any of those behaviors, depending on the starting conditions. Although these deviations are not very large for small initial amplitudes, they are still large enough so that local behavior of the motion may be dramatically changed when going from DI to DA. In addition, analysis of the Lyapunov exponent indicates (for the LHC) that the regular-motion region is considerably reduced, even if this does not lead 
directly to particle loss on our time scales. Finally; the amplitude at which an actual particle loss does take place is reduced by a similar amount, independent of whether random dipole errors are included.

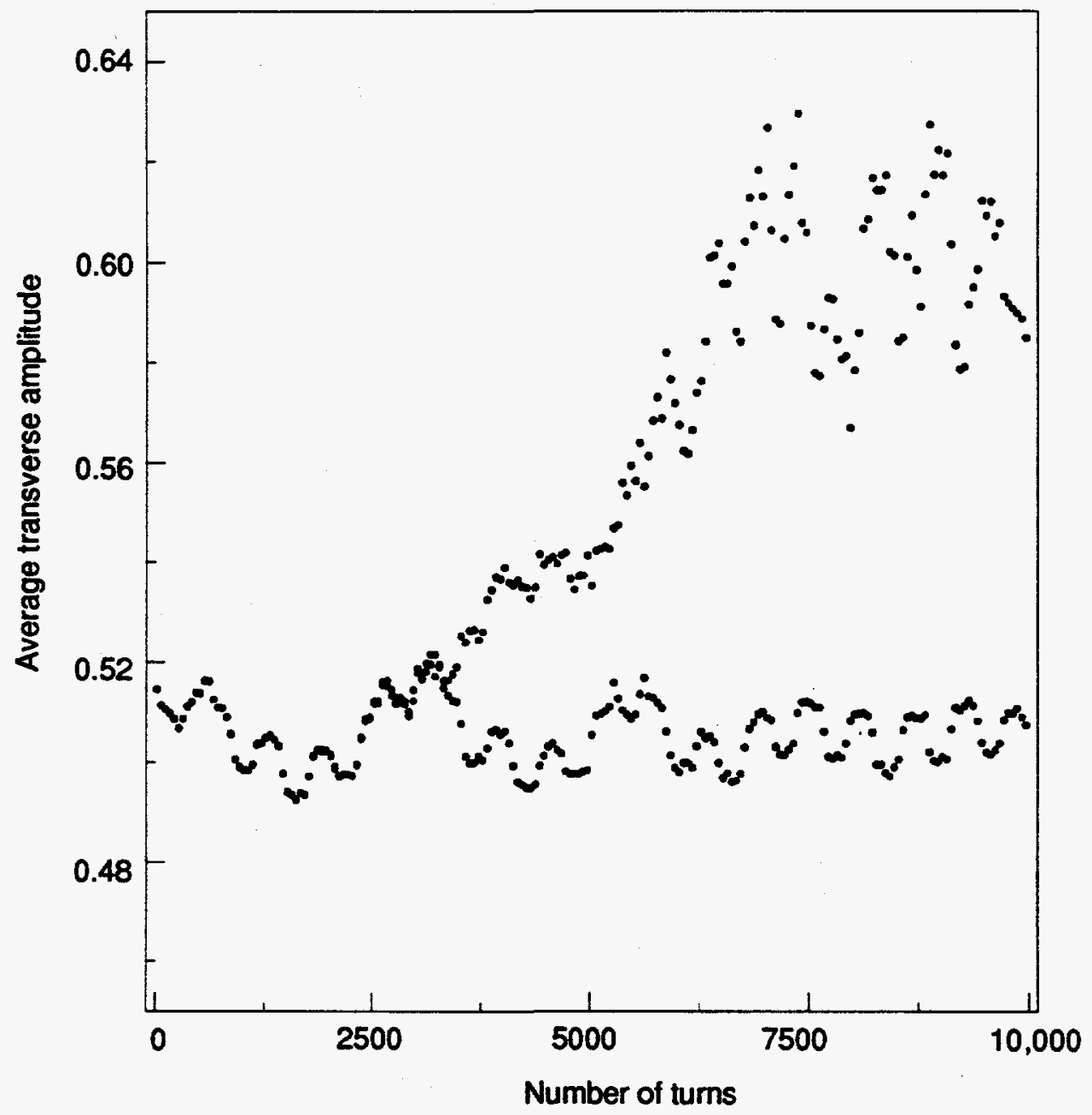

Figure 8. Amplitude Evolution as a Function of Turn Number for the SSC. For the large amplitude 0.5056 (about $18 \%$ larger than the dynamic aperture at 0.42 ) the DA results, after following the direct-tracking results for some time, suddenly diverge appreciably.

We conclude, therefore, that the differences between direct tracking and DA predictions are in all cases of practical interest sufficiently large that, in general, Taylor maps of moderate order ( $11^{\text {th }}$ order) are not guaranteed to be reliable for long-term tracking studies unless such maps are suitably symplectified. Further studies are indicated. 


\section{ACKNOWLEDGEMENTS}

We are grateful to M. Berz and É. Forest for providing us with the DA package and the Lie-algebra tools, respectively. We also thank Yunhai Cai of the SSC for his help in obtaining the nonlinear lattice. Authors Schmidt and Yan thank Mike Syphers of the SSC for his support. We also want to thank our colleagues at DESY, CERN, and SSC for helpful discussions, and Dr. H. Burkhardt for his desperately needed assistance with PAW. 



\section{REFERENCES}

1. F. Schmidt, "SIXTRACK, Version 1.1, Single Particle Tracking Code Treating Transverse Motion with Synchrotron Oscillations in a Symplectic Manner," CERN SL/90-52 (AP) (1991).

2. L. Schachinger and R. Talman, "Teapot: A Thin-Element Accelerator Program for Optics and Tracking," Part. Acc. 22 (1987).

3. M. Berz, "Differential-Algebraic Description of Beam Dynamics to Very High Orders," Part. Acc. 24 (1989).

4. S.K. Kaufmann, D.M. Ritson, and Y. Yan, "Implementation of One-Turn Maps in SSCTRK Using Zlib," SSCL Report SSCL-321 (1990); Y. Yan and C. Yan, "Zlib-A Numerical Library for Differential Algebra," SSCL Report SSCL-300 (1990).

5. Y. Yan, T. Sen, A. Chao, G. Bourianoff, A. Dragt, and É. Forest, "Comments on RoundOff Errors and on One-Turn Taylor Maps," SSCL Report SSCL-301 (1990).

6. É. Forest, M. Berz, and J. Irwin, "Normal Form Methods for Complicated Periodic Systems: A Complete Solution Using Differential Algebra and Lie Operators," Part. Acc. 24 (1989).

7. F. Schmidt, "Untersuchungen zur dynamischen Akzeptanz von Protonenbeschleunigern und ihre Begrenzung durch chaotische Bewegung," Ph.D. thesis, Hamburg University, DESY HERA 88-02 (1988).

8. F. Schmidt, F. Zimmermann, and F. Willeke, "Comparison of Methods to Determine Long-Term Stability in Proton Storage Rings," DESY HERA 91-07, CERN SL/91-14 (AP), published in Part. Acc. (1991). 